\title{
El abrigo pintado de La Hoz de Vicente (Minglanilla, Cuenca)
}

\author{
M. ${ }^{a}$ Isabel Martinez Perello . \\ Margarita diaz-Andreu Garcia *
}

\section{INTRODUCCIÓN}

El abrigo de La Hoz de Vicente, también conocido como La Rambla de Mateo, fue descubierto por don Vicente Malabia, cura-párroco de Villapardo y Villarta, junto con algunos jóvenes del primer pueblo, el 1 de mayo de 1987. La noticia fue difundida por varios diarios ', e incluso por el mismo descubridor ${ }^{2}$ pero hasta el presente no se habia publicado ningún estudio más detallado ${ }^{3}$. En marzo de 1991 realizamos una primera visita al abrigo ${ }^{4}$, tras la que nos planteamos su estudio en profundidad, dado el interés que ofrecia la gran cantidad y variedad de pinturas rupestres postpaleolíticas alli representadas.

El estudio de arte rupestre en la provincia de Cuenca se remonta al descubrimiento en 1917 del conjunto de La Peña del Escrito de Villar del Humo por O'Keliy, que se completaría por Hernández-Pacheco en 1918

* Departamento de Prehistoria e Historia Antigua, UNED.

** Department of Anthropology. University College London.

"El Día de Cuenca", 23 de julio de 1987.

Malabia, V., "De cómo descubrimos las pinturas rupestres de La Hoz de Vicente de Minglanilla (Cuenca)". Boletin de la Asociación Española de Arte Rupestre 2, 1989, págs. 25-27.

${ }^{3}$ Aunque Jesús López Requena habia realizado un trabajo no publicado en que se analizaban las pinturas con cierto detalle. Agradecemos al autor la amabilidad con la que nos brindó el material recogido. LoPez ReQuena, J., “Arte postpaleolitico en la provincia de Cuenca. Un nuevo yacimiento de pinturas rupestres: el abrigo de la rambla de Mateo, T. M. de Minglanilla", Trabajo de Doctorado, 1988, UNED. inédito.

+ En la primera visita al abrigo contamos con la eficaz ayuda de dos de los descubridores del mismo. Celestino Garcia Garcia y Eliodoro Carrasco Gandia y en la segunda con la colaboración de Alberto González. 
con la incorporación de las nuevas estaciones de la Fuente de Selva Pascuala y la Cueva del Bullón. Estos hallazgos fueron presentados en la Exposición de Arte Prehistórico Español en $1921^{5}$, pero no se publicaron de forma más detallada hasta más tarde ${ }^{6}$. Posteriormente se han realizado otros trabajos sobre este conjunto ${ }^{7}$, al que se han añadido la Peña del Castellar ${ }^{8}$, el Castellón de Los Machos ${ }^{9}$ y Marmalo ${ }^{10}$. Piñón ${ }^{11}$ publicó el abrigo de La Peña de Aldebarán de Beamud, situado más al norte dentro de La Serranía y, aunque de cronologia probablemente más moderna, se han estudiado en época reciente los grabados de El Covacho de Las Pintas de Carrascosa de La Sierra ${ }^{12}$.

La Hoz de Vicente se localiza en el extremo Nororiental de La Mancha (fig. 1). Esta zona linda por el norte con La Serrania de Cuenca, en la que hasta ahora se localizaban la totalidad de representaciones rupestres de la provincia, y por el este con la comarca valenciana de Requena-Utiel, donde no conocemos la existencia de manifestaciones artisticas de este tipo.

La topografia de la zona es accidentada. La planicie manchega llega a su fin de forma brusca en la hoz del rio Cabriel, cuyas lisas paredes profundizan el nivel del cauce hasta unos $100 \mathrm{~m}$. Más hacia el oeste la Sierra de Rubial separa esta zona de La Plana de Utiel. Son terrenos de calizas miocénicas con horizonte de humus muy poco desarrollado ${ }^{13}$, con

\footnotetext{
${ }^{5}$ Hernández-Pacheco, E., “Exposición de arte prehistórico español», Arte Español. Revista de la Sociedad de Amigos del Arte, año X, 1921, t. V, n. 7 , págs. 315-339. Madrid.

${ }^{6}$ Hernandez-Pacheco, E., Prehistoria del Solar Hispano. Memorias de la Real Academia de Ciencias Exactas, Fisicas y Naturales de Madrid, XX, 1959.

'BELTRAN, A., "Sobre la pintura rupestre levantina de un caballo cazado a lazo, del abrigo de Selva Pascuala, en Villar del Humo (Cuenca)", Miscelanea Lacarra. Zaragoza 1968. Jorda, F., "La Peña del Escrito (Villar del Humo, Cuenca) y el culto al toro", Cuadernos de Prehistoria y Arqueologia Castellonense, 2, 1975, págs. 7-9.

${ }^{8}$ Alonso, A.; Melgariejo. M.; Medina, O., y Carrion, M., "Las pinturas rupestres esquemáticas de La Peña del Castellar (Villar del Humo, Cuenca)", Zephyrus, XXXIV-XXXV, 1982, págs. 133-140.

${ }^{9}$ Alonso, A., "Los conjuntos rupestres de Marmalo y Castellón de Los Machos, Villar del Humo, Cuenca", Empúries, 45-46, 1984, págs. 8-29. Alonso, A., "Villar del Humo. Un núcleo rupestre olvidado 2, Revista de Arqueologia, 45, 1985, págs. 12-23.

${ }^{10}$ Dams, L., Les peintures rupestres du Levant Espagnol. Paris, Ed. Picard, 1984, págs. 124-128. Alonso, 1984, opus cit, nota 9. Alonso, 1985, opus cit., nota 9.

1 PIÑON, F., «Arte esquemático en Cuenca: las pinturas rupestres de la Peña de Aldebarán (Valdemoro de La Sierra)", Bajo Aragón, prehistoria, VII-VIII, 1986-87, págs. 147-157.

12 Alonso, P.; Diaz-Andreu, M.; Gonzalez, A., y Pérez, T., «El Covacho de Las Pintas (Carrascosa de La Sierra, Cuenca). Un abrigo con grabados rupestres", Revista Cuenca, 37 (en prensa).

${ }^{13}$ Guerra ef alii, en Rivas-Martinez, S., 19. Valencia. Mapa de las series de vegetación de España. Escala 1:400.000. Ministerio de Agricultura, Pesca y Alimentación. ICONA. Madrid 1985.
} 


\section{El abrigo pintado de La Hoz de Vicente (Minglanilla, Cuenca)}

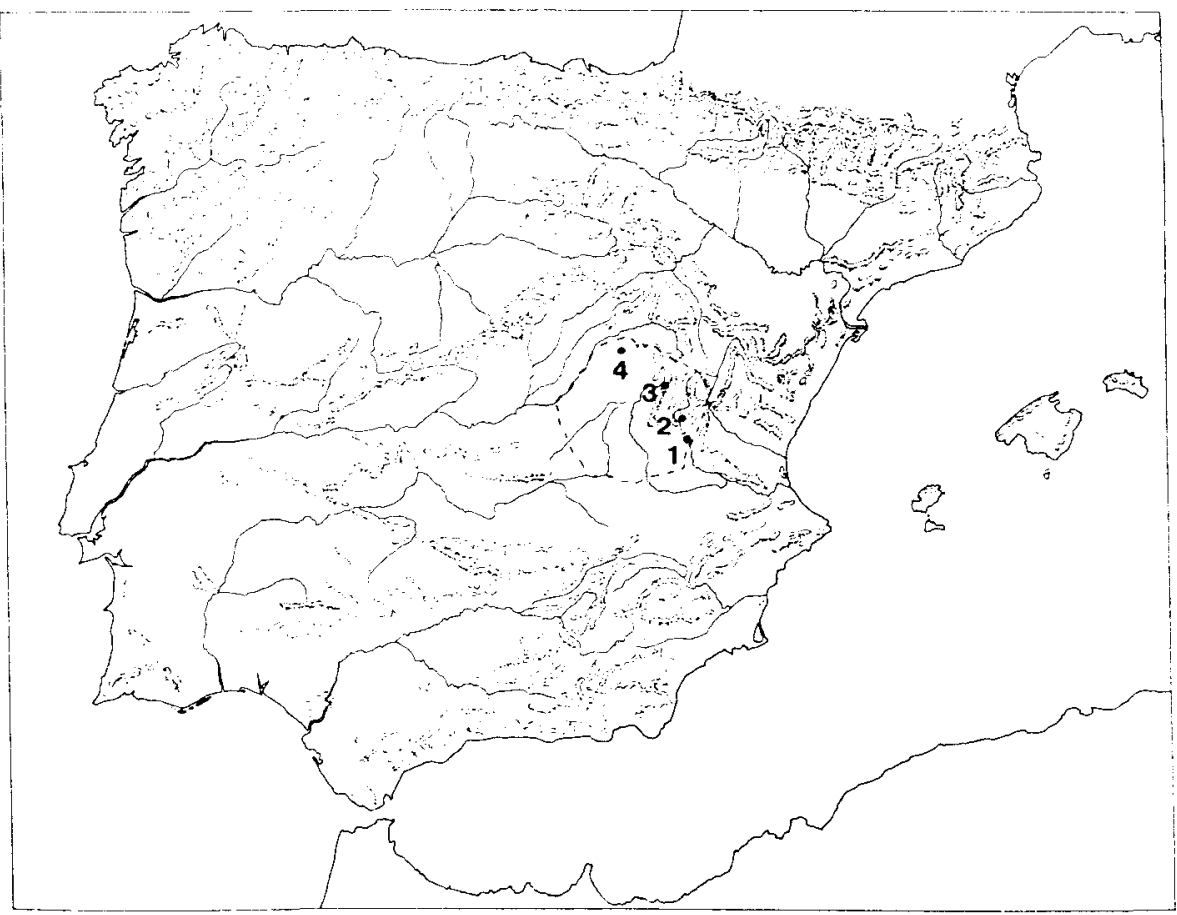

Fig. 1. Situación de las estaciones rupestres en la provincia de Cuenca. 1. La Hoz de Vicente; 2. Villar del Humo; 3. La Peña de Aldebarán; 4. El Covacho de Las Pintas.

una vegetación de piso mesomediterráneo de faciación termófila murciano-manchega con Pistacha lentiscus ${ }^{14}$. Su climatología es mediterránea genuina, moderadamente cálida seca, de inviernos frescos ${ }^{15}$.

El abrigo de La Hoz de Vicente se halla en el término municipal de Minglanilla. Se localiza en la margen derecha del rio Cabriel, en la rambla de Mateo, en las coordenadas U.T.M. 627.9 y 4371.7 , a $550 \mathrm{~m}$ de altitud ${ }^{16}$ (fig. 2). Se trata de una enorme oquedad orientada al sur a unos $20 \mathrm{~m}$ por encima del arroyo y a unos $100 \mathrm{~m}$ del cauce del rio (fig. 3). Presenta unas dimensiones aproximadas de $50 \mathrm{~m}$ de largo por $13 \mathrm{~m}$ de ancho (fig. 4) y conserva todavía restos de un muro de lo que debió ser

14 Rivas-Martinez, 1985, opus cit., nota 13

is Allue Andrade, en Rivas Martinez, 1985, opus cit, nota 13.

${ }^{16}$ Hoja número 719 (Venta del Moro) del Mapa Militar de España, escala 1/50.000. Servicio Geográfico del Ejército, 2." edición. 1979. 
un corral de ganado. La coloración oscura del terreno y los restos materiales parecen indicar la presencia de un yacimiento arqueológico en este lugar que, por la tipología de las cerámicas encontradas, podría datarse en la primera Edad del Hierro ${ }^{17}$, aunque algunas piezas liticas hagan pensar en momentos anteriores. Sin embargo, hallazgos de útiles de silex en otras cuevas no muy lejanas datadas en la segunda Edad del Hierro ${ }^{18}$ permiten suponer tambièn para éstos una cronología coetánea a los restos cerámicos.

\section{DESCRIPCIÓN DE LAS PINTURAS}

Se distinguen dos grandes zonas de pinturas, la que se sitúa en el abrigo propiamente dicho (fig. 4: zona A) y la localizada en una pared vertical a $20 \mathrm{~m}$ al oeste de la primera y orientada $150^{\circ}$ al $\mathrm{SE}$, semioculta por un derrumbe de grandes piedras (fig. 4: zona B). Cada una presenta diferentes problemas de conservación. La zona $A$ se halla gravemente deteriorada por el ennegrecimiento de la pared resultante de las hogueras realizadas por los pastores. Esta es la razón por la que las representaciones se han conservado sólo en los lugares más verticales, donde el humo no incide directamente. En la zona $B$ el deterioro viene dado fundamentalmente por el lavado o degradación de los pigmentos, producido por agentes externos y por problemas inherentes al soporte rocoso: escorrentias del agua de lluvia que ha solidificado en forma de precipitaciones de carbonato cálcico y otras concreciones calcáreas provocadas por

'El estudio mineralógico por lámina delgada de dos cerámicas procedentes del abrigo de La Hoz de Vicente, realizado en el Centro de Ciencias Medioambientales del C.S.I.C.. por Virginia Galván, presenta los siguientes resultados:

"MUESTRA 1: Matriz arcillo ferruginosa, de color anaranjado rojizo, que engloba abundantes cristales heterométricos de calcita. Parece calcita triturada mezclada con arcilla roja. La zona oscura presenta la misma composición.

En fisuras y alrededor de muchas de las inclusiones se observa un material de color negro, que parece compuesto de granos de pequeño tamaño, que pueden ser hierro no oxidado. En la masa arcillo ferruginosa destacan granillos de óxido de hierro.

MUESTRA 2: Zona clara compuesta por carbonato cálcico $\left(\mathrm{CO}_{3} \mathrm{Ca}\right)$ que engloba fósiles calizos. Se trata de una matriz arcillosa, algo rubificada, en la que destacan cristales de cuarzo y $\mathrm{CO}_{3}$ y algunos nódulos muy pequeños de óxido de hierro.

Zona oscura: matriz arcillosa de color muy oscuro, casi negro, que engloba granos de cuarzo, calcita y caliza fosilifera.

Tanto en una zona como en otra se observa heterometria en cuarzo, calcita y caliza fosilifera. Esta última es mucho más abundante que los cristales de calcita aislados.

${ }^{18}$ Martín Bonafe, M.. A., "Las cuevas del Puntal del Horno Ciego, Villalgordo del Cabriel, Valencia", Saguntum, 23, 1990, págs. 141-182. 


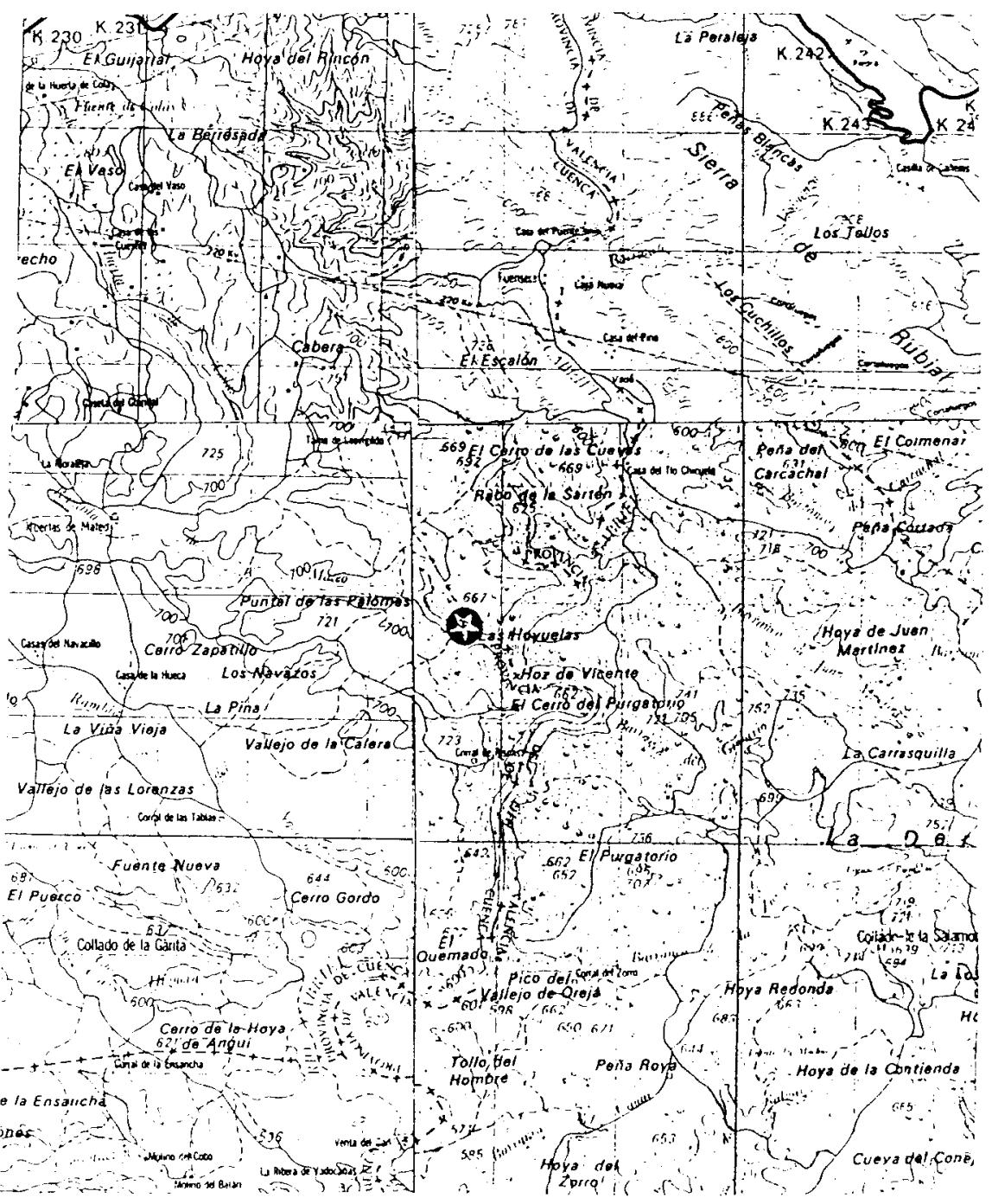

Fig. 2. Situación del Abrigo de La Hoz de Vicente.

las filtraciones y humedad de penetración por capilaridad. La exfoliación o desescamación de la corteza rocosa por cambios de temperatura o pérdida de agua de hidratación del soporte ha contribuido también a la conservación incompleta e incluso a la desaparición de motivos y conjuntos. A ello se añade la acción antrópica, ya que algunas figuras han sido picadas intencionalmente. 


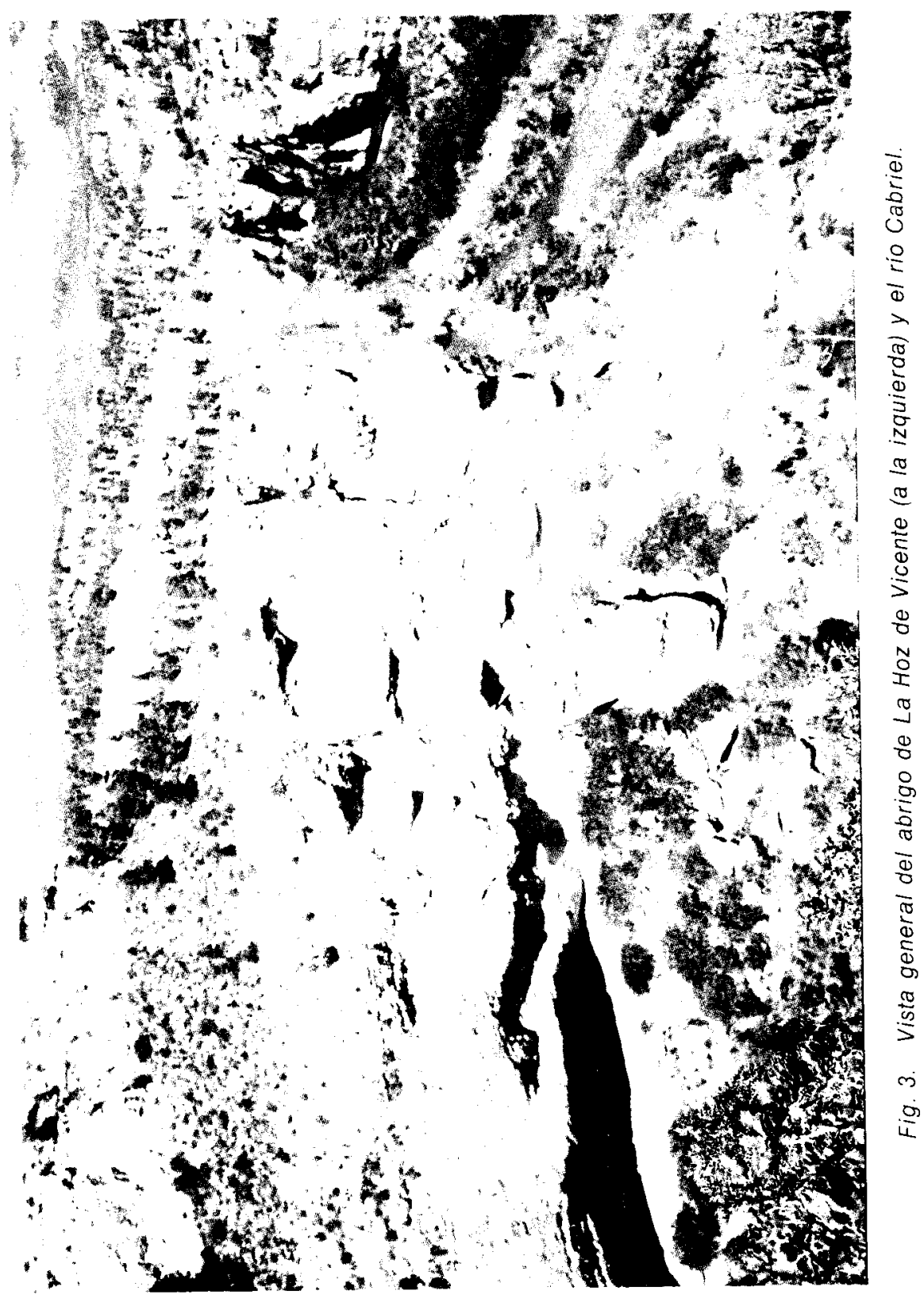



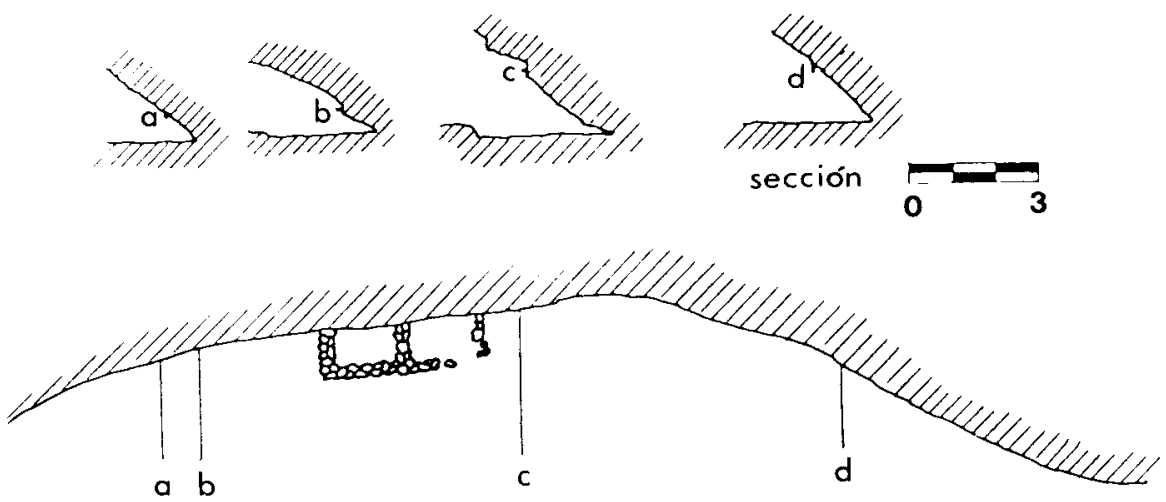

$a b$

planta

ZONA A

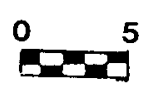

ZONA B
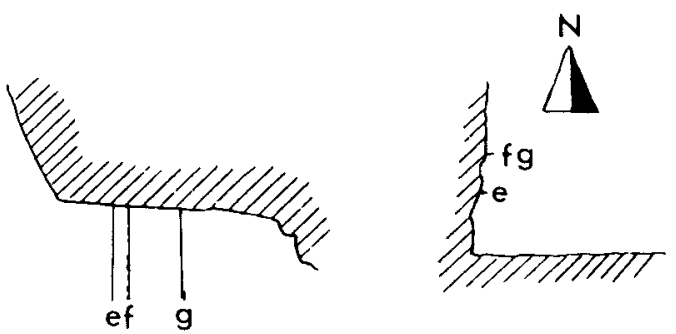

planta

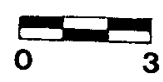

sección

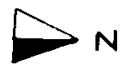

Fig. 4. Croquis de planta y secciones del abrigo, con situación de los motivos. Las secciones $A, B, C, D, E, F$ y $G$, indican la localización de los motivos de la fig. 4.1, fig. 4.2, Fig. 5, fig. 4.5, fig. 8 , fig. 6 y fig. 7 respectivamente.

En la zona $A$ se distinguen varios grupos que mantienen una homogeneidad temática, técnica y estilística. Todos ellos están realizados con un estilo naturalista, aunque en algunos se observan rasgos que revelan un cierto esquematismo. El conjunto presenta un color rojo vinoso. El tema predominante es la caza, representado por lo menos en dos ocasiones. Los escasos restos de ciervos que asimismo se conservan en el panel y la representación aislada de algún animal más completo, no nos permite asegurar la existencia de más escenas de este tipo, aunque el "ambiente» cinegético y la preocupación por la fauna salvaje resulta innegable. 
En el extremo izquierdo del abrigo a unos $60 \mathrm{~cm}$ del suelo actual, aparece representado un cuadrúpedo que interpretamos como una cabra, cuya cornamenta se traza mediante lineas rectas (fig. 5: 1). Presenta una conservación deficiente de la cabeza. Sobre ésta pequeñas manchas nos indican que tal vez poseyera, en su origen, una cornamenta más desarrollada. Su color es rojo $(189 \mathrm{u})^{19}$ y sus dimensiones son $10 \mathrm{~cm}$ de alzada y $11 \mathrm{~cm}$ de anchura ${ }^{20}$. A su izquierda, y unos centímetros más abajo, se observa una mancha indeterminada de $2,8 \times 7 \mathrm{~cm}$ del mismo color.

A $1,80 \mathrm{~m}$ hacia la derecha y a unos $50 \mathrm{~cm}$ del nivel de base, se encuentra el primer grupo propiamente dicho, formado por dos figuras, un ciervo y un arquero. La primera (fig. 5: 2) es de estilo naturalista y estilizada. Presenta una gran cornamenta y levanta las patas delanteras en actitud dinámica, ofreciendo éstas un tamaño muy reducido en comparación con lo voluminoso del cuerpo del animal, aunque esta desproporción no disminuye la armonia de la representación. Muestra un color granate algo diferente al resto de las representaciones, siendo su tonalidad más oscura (color $1815 \mathrm{u}$ ). Sus dimensiones son de $7 \times 24 \mathrm{~cm}$. Su conservación es deficiente, al encontrarse bastante diluida la pigmentación de la parte posterior del animal. Frente a éste se encuentra una figura humana más estilizada e idealizada (fig. 5: 3), armada con un gran arco de curvatura poco pronunciada y dirigiendo una flecha hacia las patas del animal. Dos trazos que parten de la línea vertical que forma el tronco de la figura y que se unen en su extremo, podrian interpretarse como un carcaj. Además parece observarse que el inviduo lleva una especie de “bolsa» (?) junto a las piernas. Su color es idéntico al del ciervo y sus dimensiones son de unos $11,5 \times 5,5 \mathrm{~cm}$ (fig. 6). Junto a estas dos representaciones se observan manchas indeterminadas de pintura a derecha e izquierda, e incluso debajo del ciervo. La de mayor tamaño se localiza a $6 \mathrm{~cm}$ a la izquierda del arquero y constituye un motivo formado por una línea vertical de unos $4 \mathrm{~cm}$ de largo que en su extremo superior se remata con un pequeño trazo horizontal hacia la derecha y en el inferior con otro recto y perpendicular al eje vertical. La anchura del trazado es de unos $29 \mathrm{~cm}$ y su color similar al de los dos motivos descritos.

A un metro a la derecha se encuentran restos de un ciervo y a otro metro más a la derecha se localizan tres barras y varios trazos indeterminados.

19 Para la determinación del color hemos empleado la tabla cromática Pantome Color Formula Guide, 5th Printing, 1988-89, Pantome inc., New Jersey, USA. Los colores se tomaron en primavera, a mediodia y con el soporte húmedo.

${ }^{20}$ Las dimensiones de las figuras se expresan en el orden siguiente: altura y anchura máximas. 


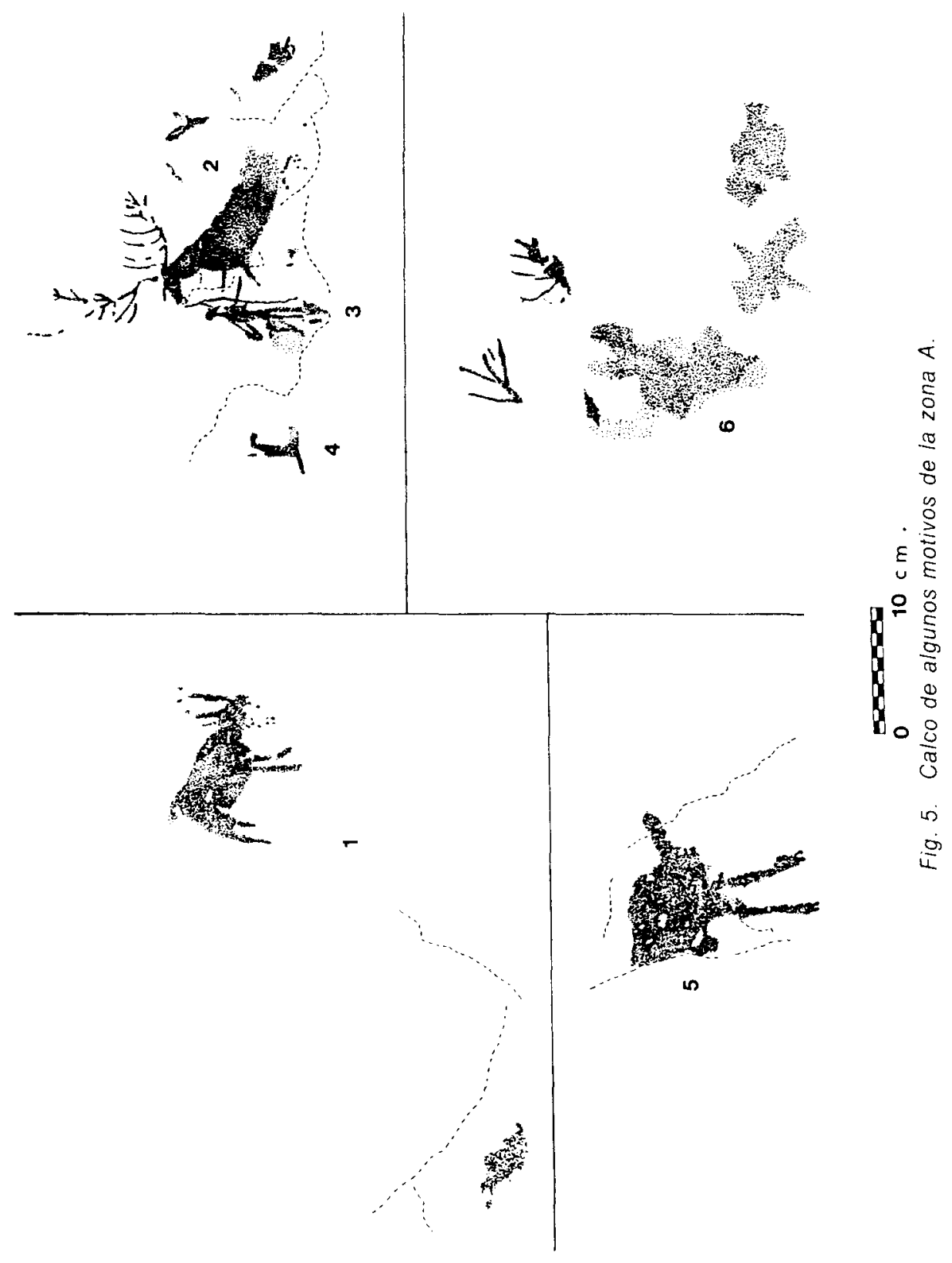




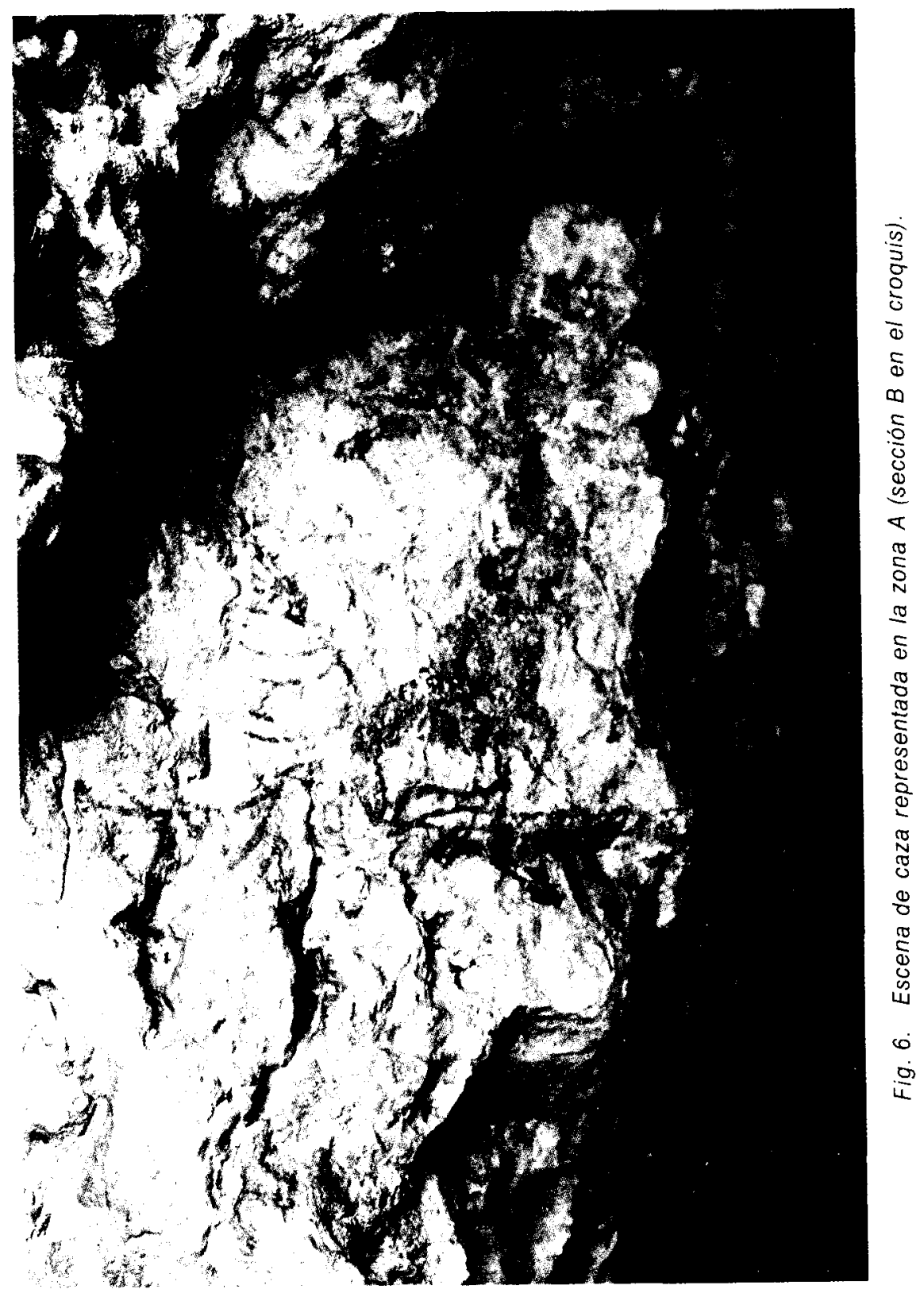


Al otro lado del muro del corral derruido y a 1,36 m del suelo, aparece un conjunto formado por un ciervo seminaturalista rodeado por dos arqueros de estilo bastante esquemático (fig. 7), todos con el mismo tono rojo vinoso. La primera figura, de unas dimensiones de unos $29 \times 40 \mathrm{~cm}$, muestra una gran cornamenta bastante bien conservada, mientras que el cuerpo del animal está afectado por la pérdida de pigmentación, desprendimiento de la superficie rocosa y la existencia de concreciones calcáreas. Presenta un cierto dinamismo reflejado en el movimiento de avance de sus patas, en las que se detallan las pezuñas. Es interesante resaltar cómo el autor aprovecha cierta oquedad en el relieve de la roca para marcar el volumen del cuerpo del animal. Conserva un tono rojo vinoso (172 u) que se oscurece en la parte trasera (1815 u). El animal agacha la cabeza y la dirige hacia un primer arquero situado a unos $6 \mathrm{~cm}$ a la derecha. Éste se halla constituido por una fina linea vertical que presenta un ligero ensanchamiento en el extremo superior para indicar la cabeza, y una bifurcación de su extremo inferior, con trazos más delgados, a modo de piernas en ángulo. Éstos se rematan con lineas cortas y horizontales que indicarían los pies en posición de avance, detallando en uno de ellos tres dedos. Del torso parte un tramo semicircular que interpretamos como un arco, cuya cuerda estaria representada por una línea vertical paralela a la del tronco del arquero. Este arco está atravesado por dos líneas perpendiculares, de las que la inferior señalaria el brazo izquierdo sujetando el arma y la superior plasmaria el brazo derecho sosteniendo la flecha que se dirige hacia el ciervo, y en cuya punta se dibujan tres trazos cortos. Las dimensiones de esta figura son de unos 23,5 $\times 15,5 \mathrm{~cm}$ (fig. 8). El segundo arquero, de dimensiones $(25 \times 12 \mathrm{~cm}$ ) y color (172 u) semejantes al anterior, se sitúa a $38 \mathrm{~cm}$ a la derecha del animal. Su constitución es muy similar a la del primero, aunque éste dirige su flecha hacia los cuartos traseros del cuadrúpedo. El estado de conservación de esta figura humana es deficiente dado lo perdido de los trazos que la constituyen. El grupo forma una composición simétrica en la que el eje central es el objeto de caza, un ciervo de bastante edad a juzgar por lo desarrollado de su cornamenta.

A $65 \mathrm{~cm}$ a la derecha de este conjunto y a 1,15 $\mathrm{m}$ del suelo se hallan los restos de una nueva cornamenta de un ciervo (fig. 5: 6), bajo la que se observan algunos restos poco definidos de pintura que corresponderian al cuerpo del animal. Su color es rojo vinoso (179u).

En el extremo derecho de la zona $\mathrm{A}$, a unos $13 \mathrm{~m}$ del anterior motivo y a 1,30 $\mathrm{m}$ del suelo, se observan los restos de un animal (ciervo o cabra) del que sólo se han conservado los cuartos traseros, indicando las patas con pezuña y el rabo (fig. 5: 5). Esta figura presenta unas dimensiones de $12 \times 12 \mathrm{~cm}$. 

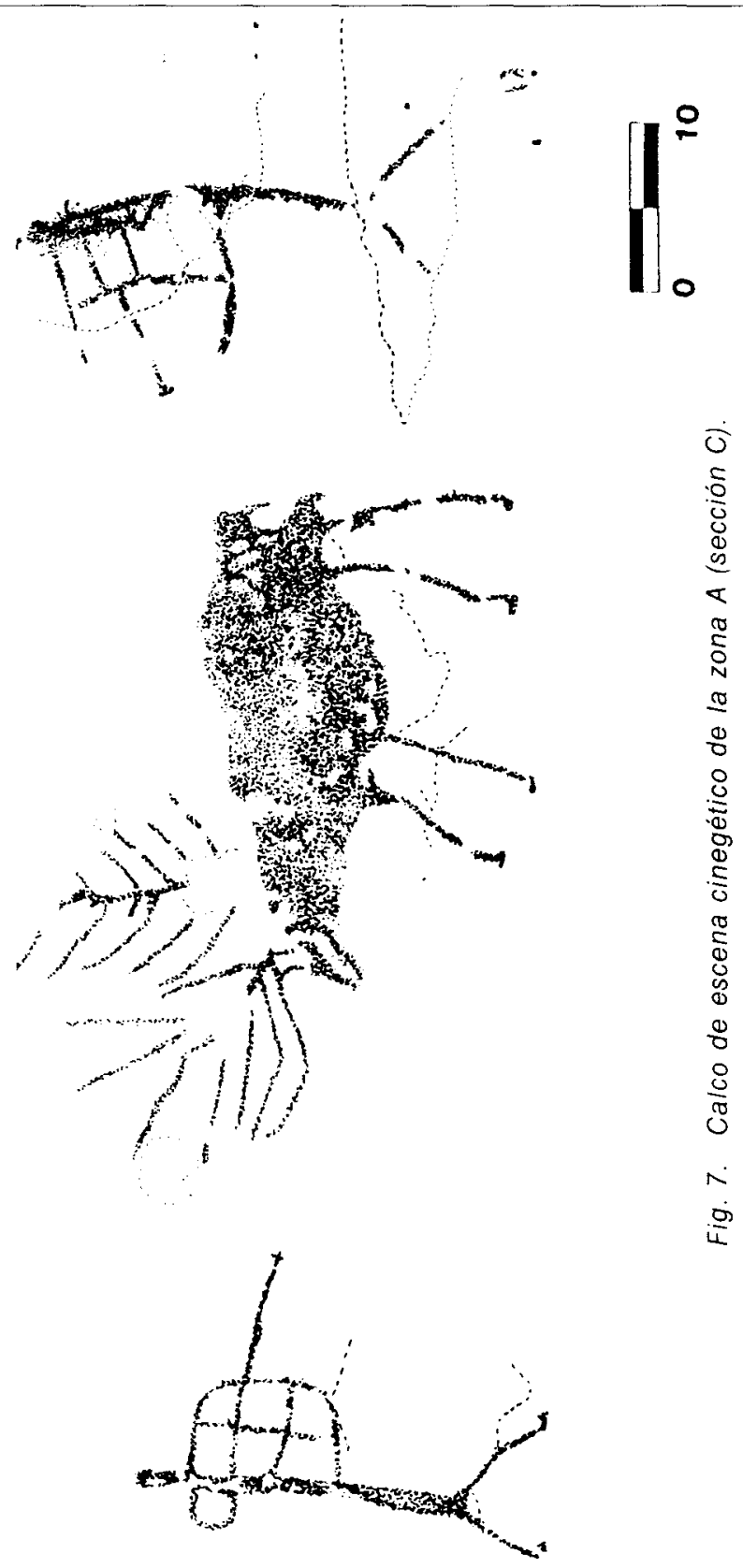


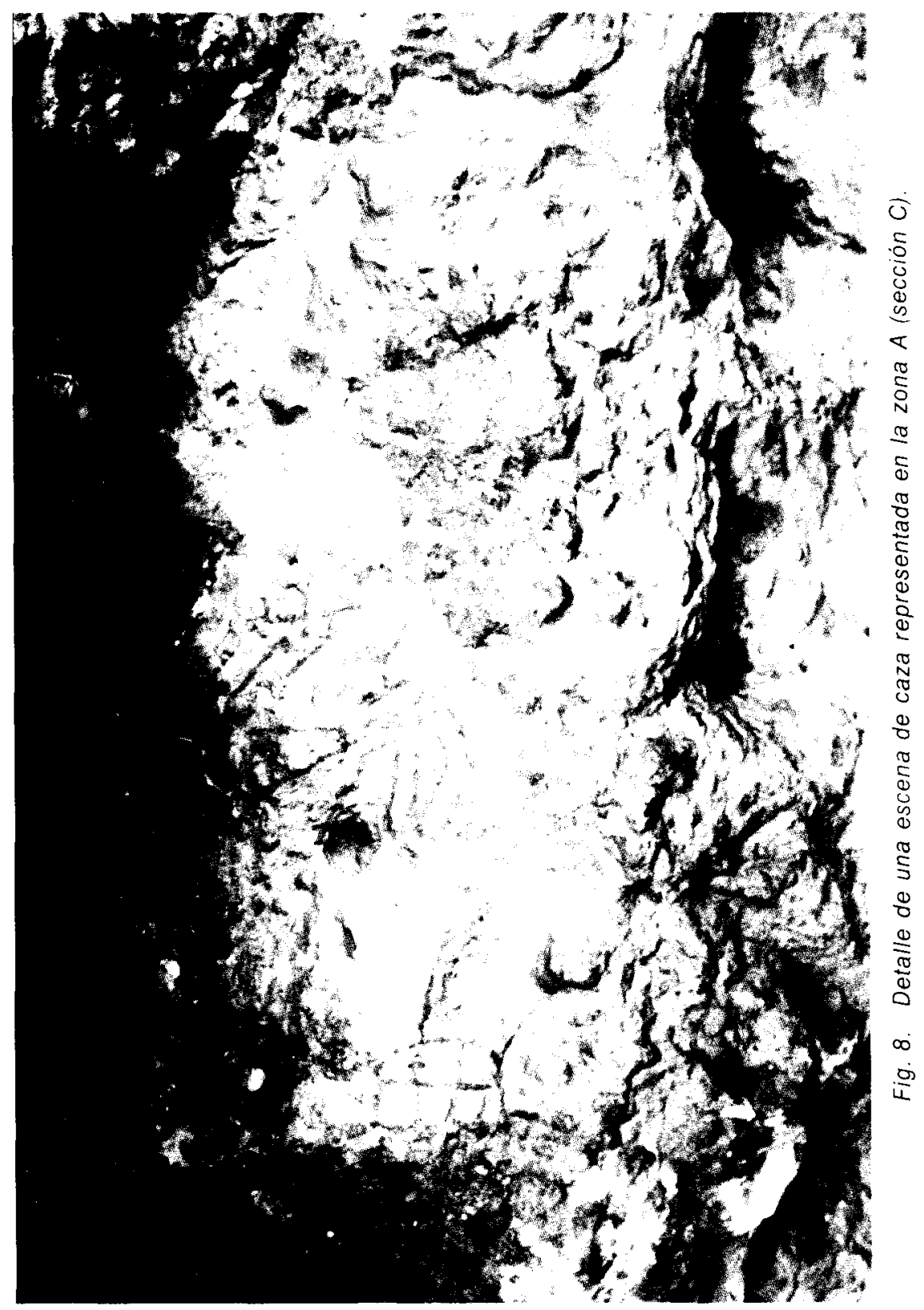


Las pinturas de la zona $B$ se sitúan sobre una pared vertical de unos $10 \mathrm{~m}$ de alto y $5 \mathrm{~m}$ de ancho, orientada al SE y de aspecto liso. Como en la zona $A$, el color predominante de las pinturas es el rojo vinoso. Sin embargo aquí no se observa la mismo homogeneidad de estilos, puesto que se combinan las figuras de estilo naturalista con las de tendencia esquemática y las claramente esquemáticas. La temática también presenta una mayor variedad, aunque faltan las escenas de caza mayoritarias en la zona $A$. En la descripción se sigue en lo posible un orden de izquierda a derecha y de arriba a abajo.

En el primer grupo de motivos (figs. 9 y 10) destacan dos grandes figuras humanas de estilo naturalista de desigual conservación, cuya relación parece indudable. La de mayor tamaño (fig. 9: 1) $(49 \times 22 \mathrm{~cm}$ ) presenta un trazo vertical que se ensancha en su extremo superior para indicar el torso triangular. Sobre él se representa la cabeza en la que se detallan varios trazos rectos y oblicuos que se podrian interpretar como cabello, penacho u otro tipo de tocado. El brazo derecho del sujeto forma un arco semicircular que pasa por encima del tronco a la altura de la cintura, mientras que el izquierdo, muy incompleto y en el que se detalla la mano, sujeta un arco, del que sólo quedan algunos trazos. Estos son suficientes para poder apreciar sus grandes dimensiones, que igualarian en altura a las del individuo. De las piernas sólo quedan restos de una linea vertical prolongación del tronco. Su color es rojo vinoso (180-181 u), en lo que se asemejan al resto de los motivos del panel, salvo las excepciones que detallaremos. A un par de centímetros a la izquierda se localiza el segundo personaje (fig. 9: 2), del que se han conservado la cabeza, que parece tocada con un penacho, y algunos restos poco definidos del tronco y brazos cuyas dimensiones son $29 \times 9 \mathrm{~cm}$. A esta figura parece asociarse un conjunto de lineas convergentes que se sitúa entre ambas representaciones humanas y que interpretamos como un haz de flechas que portaria este segundo individuo. Nos hallariamos asi ante un arquero principal y su acompañante porteador. Se superpone a la última representación descrita un motivo antropomorfo de estilo netamente esquemático de trazo mucho más grueso, de $19 \times 12 \mathrm{~cm}$ (fig. 9: 3). Está constituido por un eje vertical que hace de cabeza, tronco y sexo, interrumpido arriba y abajo por trazos en arco indicando brazos y piernas. A la derecha del arquero se ubican dos nuevos antropomorfos similares al descrito pero incompletos (fig. 9: 4), de $7 \times 9,5$ y $13 \times 17 \mathrm{~cm}$ respectivamente. También superpuesto, pero ahora sobre el extremo inferior del arquero, se encuentra una pequeña figura esquemática antropomorfa de tipo cruciforme (fig. 9: 5), indicando las piernas con un trazo horizontal y una cabeza circular en la que sólo se perfila el contorno. Sus dimensiones son unos $7,5 \times 6 \mathrm{~cm}$ y su color, muy oscuro, se aproxima al negro. 

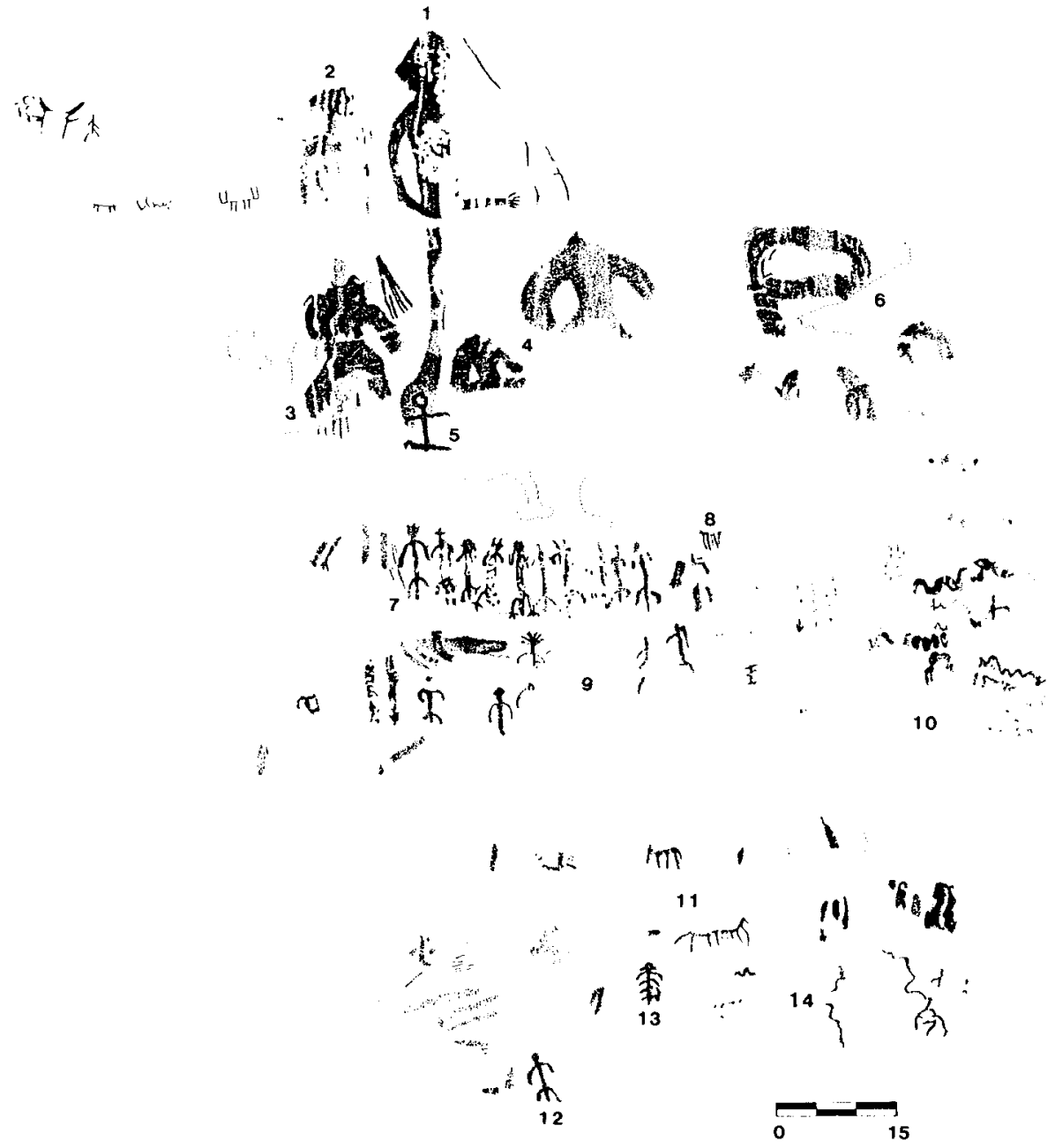

Fig. 9. Calco del extremo superior izquierdo del panel de la zona B (sección F). 


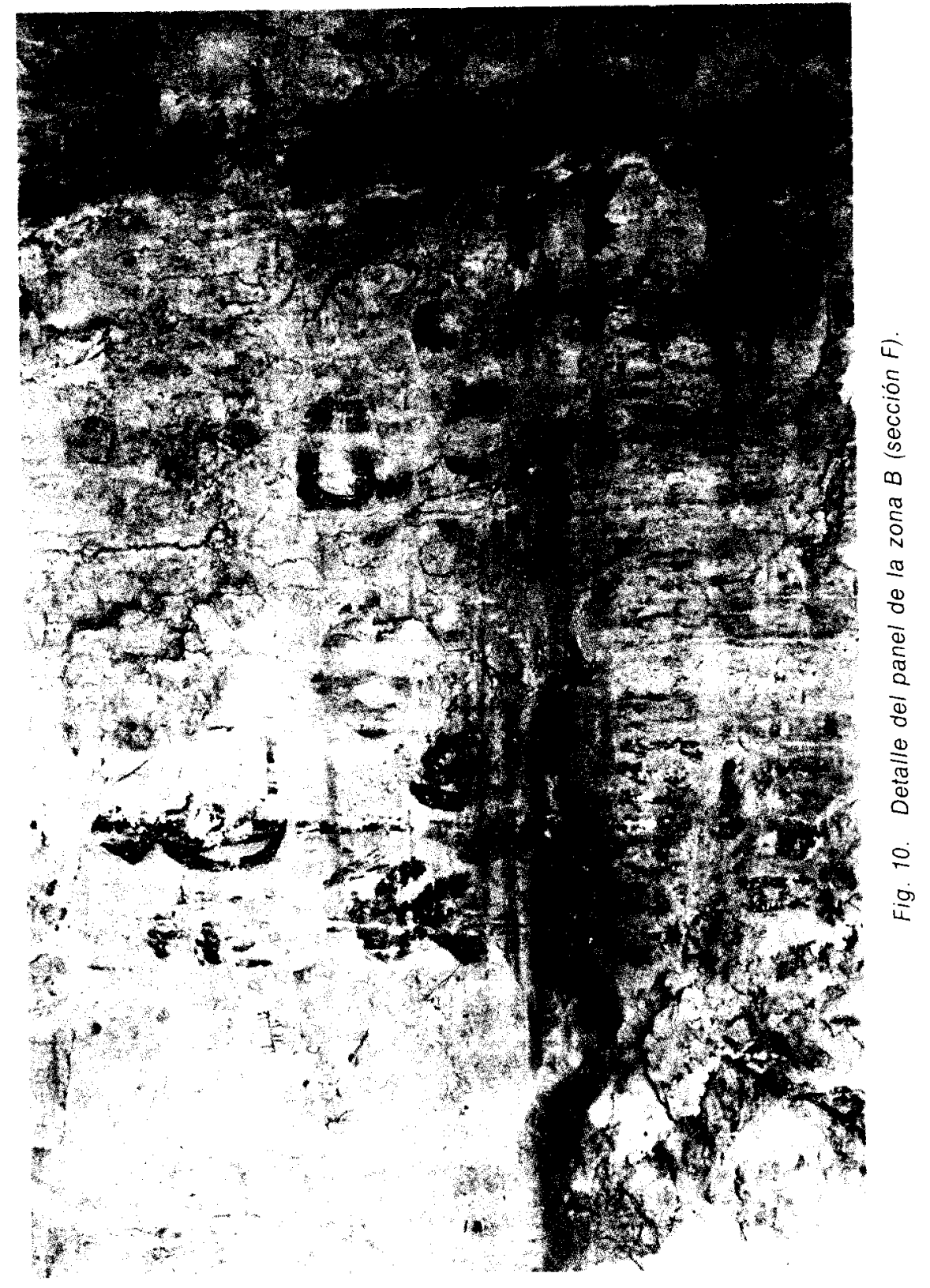


A $22 \mathrm{~cm}$ a la derecha del arco antes mencionado aparecen restos de pintura de un grueso trazo vertical del que parte a la derecha un tramo semicircular formando un espacio interior ovalado de unos 13,5 $\times$ $15,5 \mathrm{~cm}$ (fig. 9: 6). Bajo esta figura, y a su derecha, se observan algunas pequeñas manchas de trazo curvo.

A algo más de medio metro de la figura anterior y a $1,28 \mathrm{~m}$ del suelo se encuentra un conjunto de motivos en los que se evidencia la superposición de figuras (figs. 11 y 12). Parecen ser de un primer momento los numerosos restos de pintura dispuestos en pequeñas agrupaciones verticales que en ocasiones podrian corresponder a antropomorfos, de un tamaño aproximado de $20 \mathrm{~cm}$ de longitud y $3 \circ 4 \mathrm{~cm}$ de anchura. A algunos de estos motivos se superponen dos (fig. 11: 1) de las nueve figuras masculinas del tipo de brazos y piernas en arco, de $11 \times 5 \mathrm{~cm}$ de tamaño medio y un único posible zoomorfo (fig. 11: 2), de $10 \times 3,5 \mathrm{~cm}$, motivos que se distribuyen por todo el panel. El estado de conservación de este grupo es variable ya que el friso está muy afectado por las concreciones calcáreas y alguna de las figuras muestran repiqueteados de origen antrópico. En general mantienen una coloración en rojo vinoso oscuro (195 u).

A unos $10 \mathrm{~cm}$ bajo las dos grandes figuras naturalistas ya descritas se localiza un conjunto de die (quizá once) figuras antropomorfas masculinas esquemáticas alineadas horizontalmente (fig. 9: 7). Todas presentan dimensiones (unos $10 \times 2,5 \mathrm{~cm}$ ), estilo y técnica similares, en las que se destacan la representación de detalles anatómicos, como los dedos de los pies y tocados sobre la cabeza. Algunas mantienen los brazos alzados y flexionados en posición de orante, llegando casi a entrelazarse. La primera de ellas parece portar un objeto alargado formado por tres finas lineas, quizá un arco o un bastón de color más claro, y a su izquierda se localizan cuatro barras más gruesas, acaso restos de otras figuras humanas. A la derecha de la última unos trazos podrian corresponder a una nueva figura antropomorfa sobre la que se dibuja un pequeño motivo pectiniforme, quizá una zoomorfo, de unos $2,5 \times 3 \mathrm{~cm}$ (fig. 9: 8).

Bajo la línea de antropomorfos se localizan restos de cinco nuevas figuras humanas (fig. 9: 9). Dos de ellas muestran sendos tocados, uno en forma de penacho y otro de «boina», de $10 \times 3,5$ y $6,5 \times 3 \mathrm{~cm}$ respectivamente. Dado el carácter incompleto de las figuras, no queda claro, salvo en un caso, su adscripción sexual. El conjunto contiene además varias manchas de pintura, que proliferan sobre todo el extremo derecho, en el que se observan digitaciones y varios serpentiformes o restos de ellos de 1 ó $0,5 \mathrm{~cm}$ de grosor y 12 ó $9 \mathrm{~cm}$ de longitud (fig. 9: 10). Desgra- 

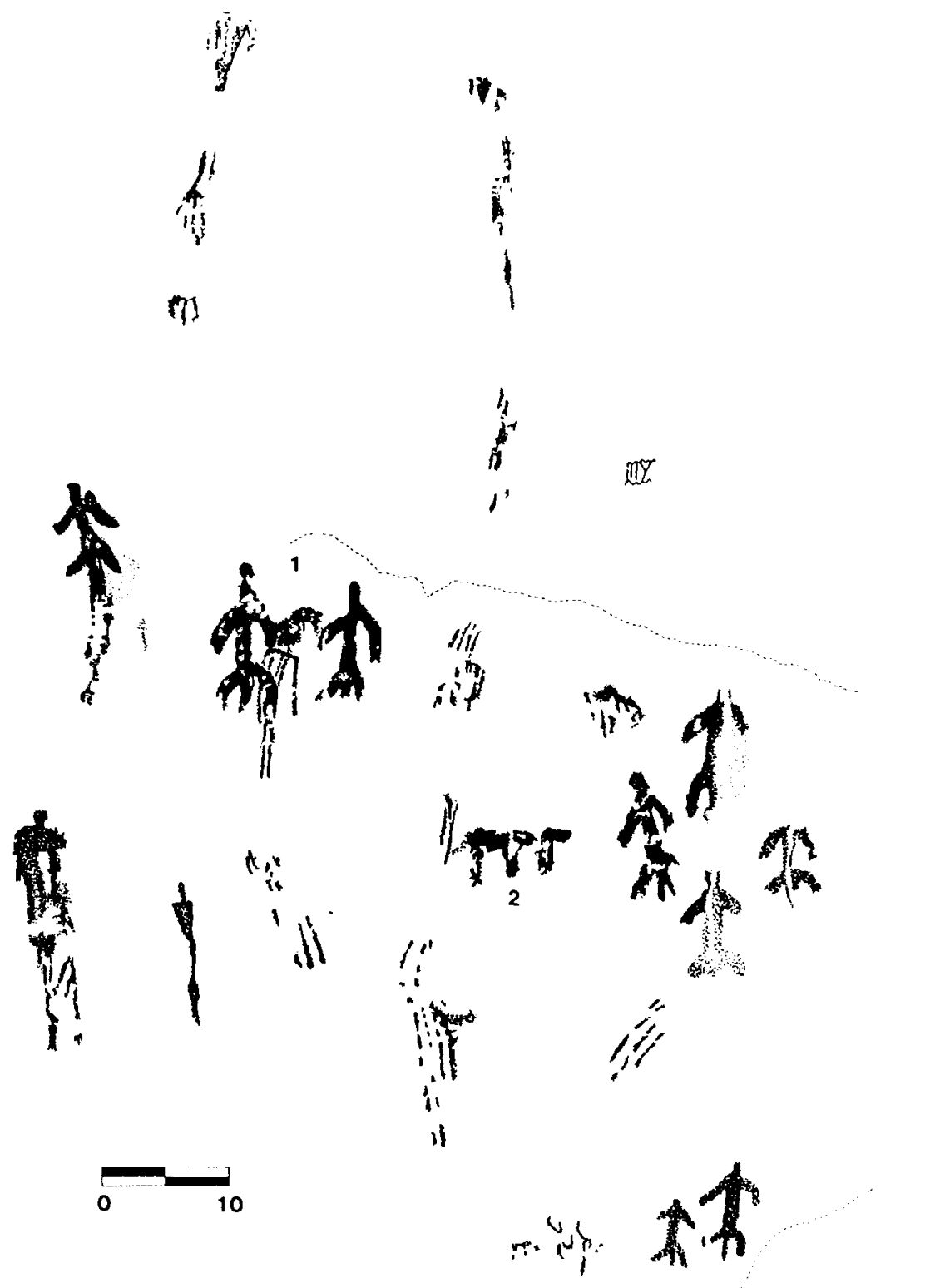

Fig. 11. Calco del extremo superior derecho de la zona $B$ (sección G). 


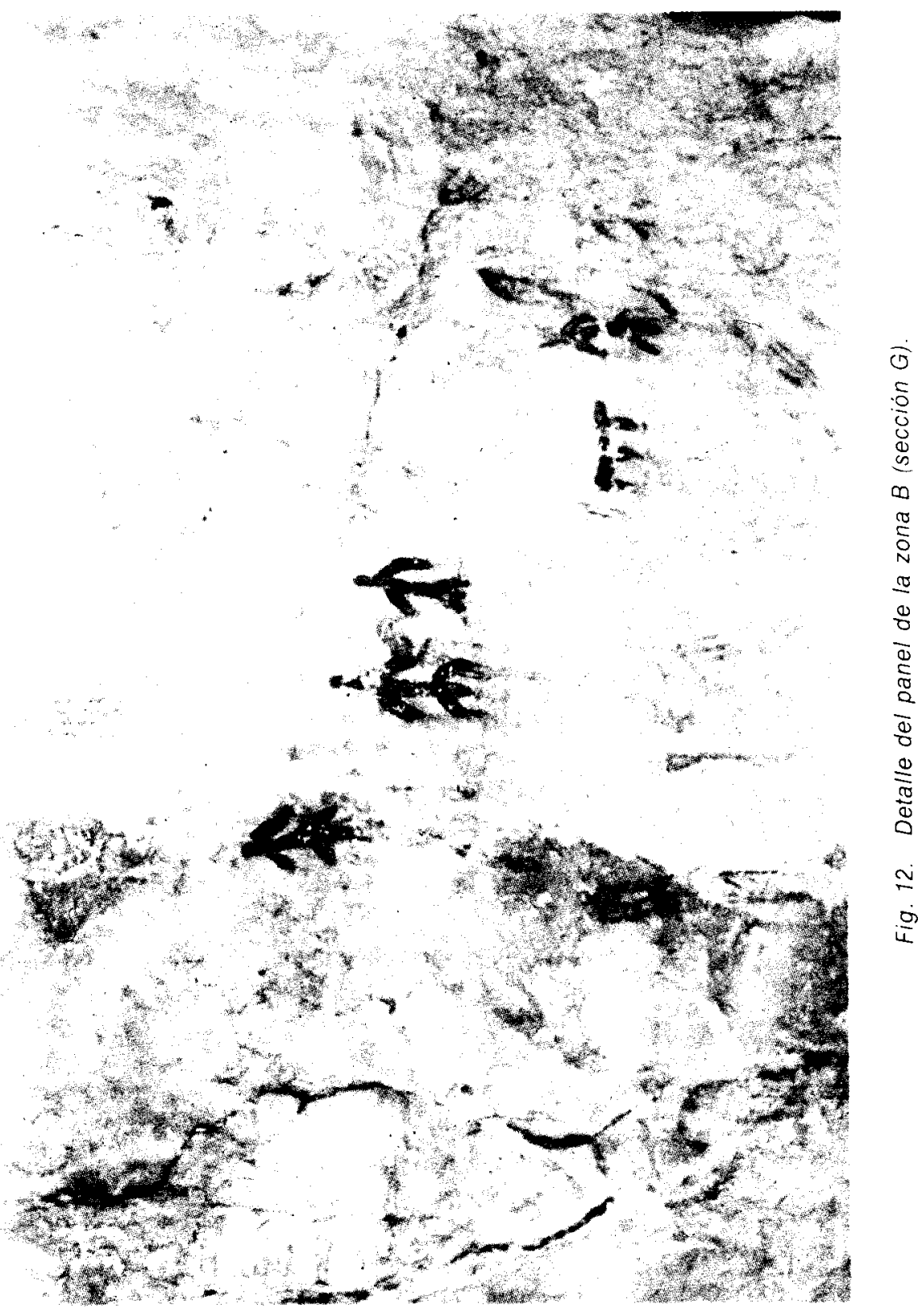


ciadamente las concreciones calcáreas han cubierto la mayor parte de este grupo de pinturas, por lo que su lectura resulta problemática.

A unos $30 \mathrm{~cm}$ bajo el grupo de antropomorfos alineados se encuentran varias siluetas aparentemente inconexas de marcado estilo esquemático. Destacan dos representaciones pectiniformes, posiblemente zoomorfos, de $3 \times 5$ y $10 \times 2,5 \mathrm{~cm}$ cada una (fig. 9: 11). Además se observan un antropomorfo masculino del tipo de brazos y piernas en arco, de $7 \times$ $3,5 \mathrm{~cm}$ (fig. 9: 12), un ramiforme, de $5,4 \times 3,4 \mathrm{~cm}$ (fig. 9: 13), varios trazos ondulantes o serpentiformes (fig. 9: 14), barras y restos indeterminados.

Bajo un saliente de la roca, a $60 \mathrm{~cm}$ bajo la linea de antropomorfos descrita, se representa una escena (figs. 13 y 14) en la que un conjunto de al menos trece cuadrúpedos de cuerpo lineal y cuernos y patas en perspectiva ligeramente torcida, que definiriamos como cabras, de los que el mayor mide unos $5,7 \times 9,5 \mathrm{~cm}$ (fig. 13: 1), siguen a una figura humana tocada con penacho (fig. 13: 2), de unos $7,5 \times 3 \mathrm{~cm}$. A $10 \mathrm{~cm}$ a la derecha se dibuja una complicada silueta (fig. 13: 3). Parece tratarse de una figura antropomorfa, de $7 \times 7 \mathrm{~cm}$, tocada con grandes cuernos, que sujetaría con su mano derecha un serpentiforme. Lamentablemente la parte inferior de la figura no queda muy bien definida debido a su mala conservación, pero se observan varios trazos ondulantes. Bajo esta figura todavia se observan manchan indeterminadas y lo que parecen dos zoomorfos (el más evidente con dimensiones de $2 \times 3,5 \mathrm{~cm}$ (fig. 13: 4) y dos antropomorfos de $5 \times 3,5$ y 6,3 $\times 2,8$ respectivamente (fig. 13: 5). A su izquierda y bajo el rebaño descrito se halla una representación de interpretación dudosa y tendencia poligonal, de unos $4,3 \times 4 \mathrm{~cm}$, a la que se asocia un cuadrúpedo de unos $3 \times 5 \mathrm{~cm}$ (fig. 13: 6). Bajo estos motivos encontramos una mancha indeterminada de $3 \times 7,5 \mathrm{~cm}$ y forma triangular (fig. 13: 7).

Quedan por describir unas figuras (fig. 9: 15) situadas en el extremo superior izquierdo de todo el conjunto de representaciones de la zona B. Si lo hacemos en último lugar es por parecernos que su cronologia es mucho más reciente. Se representan con fino trazo y un color negro similar al producido por un lápiz de grafito, un conjunto formado per dos antropomorfos, un zoomorfo, líneas en zig-zag y varios signos, todos ellos de reducido tamaño y de marcado estilo esquemático.

\section{EL ABRIGO DE LA HOZ DE VICENTE EN EL CONTEXTO PENINSULAR}

El abrigo de La Hoz de Vicente contiene más de un centenar de representaciones rupestres que muestran diferentes estilos y temática, aun- 


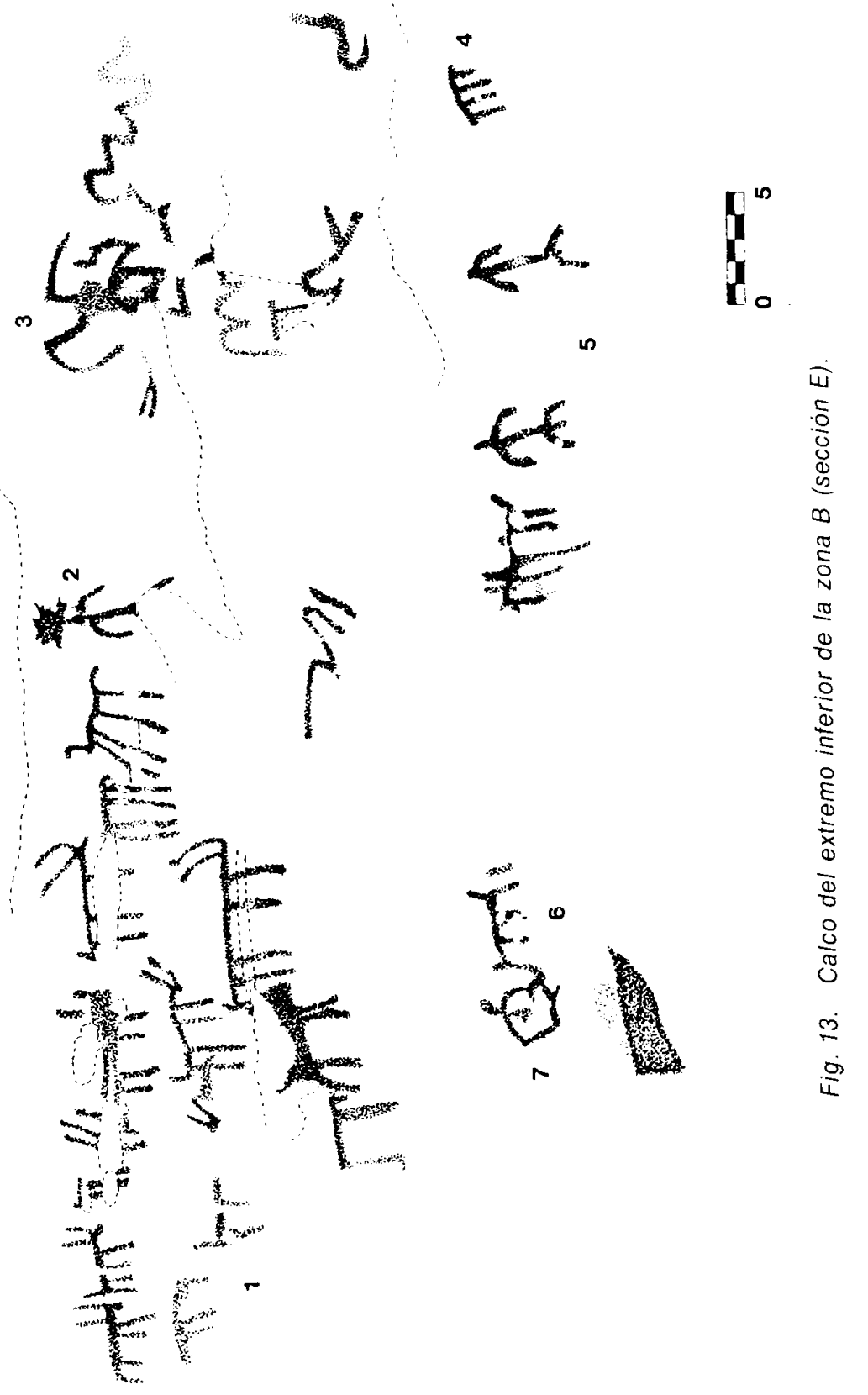



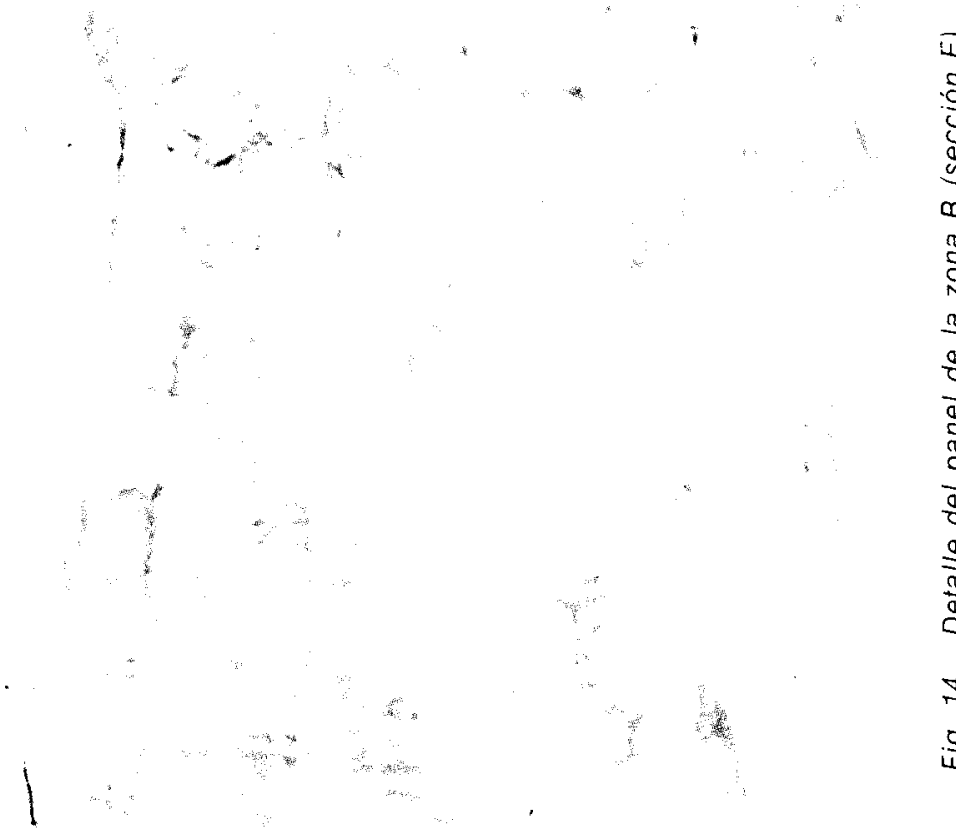
$\dot{I}$

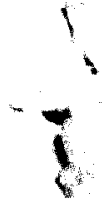


que una similar "técnica pictórica". Todas las figuras se han realizado con tintas planas y son monócromas, predominando el color rojo vinoso. Se han aprovechado las zonas verticales para su plasmación, caracteristica más marcada en la zona $A$, donde sólo se observan pinturas en los escasos sitios donde la roca cumple este requisito, aunque el ennegrecimiento del resto del panel debido a las hogueras realizadas por los pastores podría ocultar otros motivos situados en planos oblicuos.

Se distinguen varios estilos: naturalista, naturalista-estilizado, semiesquemático y esquemático, que no se reparten de forma homogénea en las dos zonas, ya que los dos primeros se localizan en la zona A, y los dos últimos son mayoritarios en la $\mathrm{B}^{21}$.

Los temas representados son antropomorfos, zoomorfos y otras figuraciones más abstractas. Los motivos antropomorfos se plasman con diferentes estilos, desde el naturalista hasta el esquematico. La mayoria presentan el atributo sexual masculino, sin que podamos asegurar que las figuras afálicas sean representaciones femeninas. En la zona A se localizan tres arqueros (fig. 5: 3 y 5), todos ellos en disposición de disparar, pero sólo uno de ellos ofrece un estilo naturalista estilizado. Éste porta un elemento sobre su espalda para el que no conocemos paralelos en la Península lbérica. Los antropomorfos de estilo naturalista localizados en la zona B son dos. Son éstas las figuraciones de mayor tamaño de todo el conjunto (fig. 9: 1 y 2). El arquero tiene su paralelo más cercano por la forma de su arco y el modo en que lo sujeta en el abrigo de Selva Pascuala de Villar del Humo ${ }^{22}$ y por su tamaño en todo el área de Teruel, Castellón, Valencia, Albacete y Murcia ${ }^{23}$. Presenta un tocado o peinado para el que encontramos paralelos en la cueva de La Vieja (Alpera) ${ }^{24}$ y en Solana de Las Covachas (Nerpio) ${ }^{25}$. Por otra parte, en el abrigo del

\footnotetext{
"Hemos optado por utilizar el término "semiesquemático" para una diferenciación a nivel metodológico, a fin de distinguir ciertas figuras en las que se plasman detalles somáticos, pero cuyo grado de estilización las aproxima al arte esquemático. Sin embargo. coincidimos con Jordá (nota 22, pág. 129) en que "son consecuencia del mismo proceso esquematizador, dificil de valorar con criterios objetivos, ya que no existen normas generales y válidas que nos aseguren cuándo empieza lo seminaturalista y en qué momento se realiza el paso a lo esquemático". Jonda, F.: Historia del Arte Hispánico, 1. La Antiguedad, 1. Madrid, Ed. Alhambra, 1978.

"Beltran, A., Arte rupestre levantino, Monografias Arqueológicas, IV. Zaragoza 1968. pág. 159.

${ }^{23}$ Dams, 1984, cit. nota 10, págs. 1-6 y 10, y fig. 198.

${ }^{24}$ Beltran 1968, cit. nota 22, pág. 235.

25 Alonso, A., El conjunto rupestre de Solana de Las Covachas, Nerpio (Albacete). Albacete, Instituto de Estudios Albacetenses, 1980, pág. 142, y fig. 153
} 
Ciervo de Dos Aguas (Valencia) ${ }^{26}$, dos arqueros muestran, igual que esta figura, una mano en la que indican los dedos, aunque en aquéllos ésta no sujeta el arco. En Solana de Las Covachas ${ }^{27}$ también se representan los dedos en la mano de un arquero. Los haces de flechas, como el que parece portar el segundo individuo (fig. 9: 2), son habituales en el arte levantino, aunque lo más frecuente es su representación en horizontal, como por ejemplo en el Civil de la Valltorta (Castellón) ${ }^{28}$ y La Solana de Las Covachas (Albacete) ${ }^{29}$. Figuras de este tipo encontramos también en el abrigo de El Arquero de Santolea (Teruel) ${ }^{30}$ y de estilo similar en su tocado de estructura algo triangular, muy frecuente en el arte levantino, en el Abrigo de La Sola de Las Covachas (Albacete) ${ }^{31}$ y en la Fuente del Sabuco (Murcia) ${ }^{32}$.

Entre las figuras antropomorfas esquemáticas se distinguen diversos tipos, según la tipologia de Acosta ${ }^{33}$ : de brazos y piernas en arco o ángulo, cruciformes y ramiformes. El primero es mayoritario, lo que es habitual en el arte rupestre esquemático de la Peninsula Ibérica, con ejemplos próximos en el abrigo de la Peña del Castellar de Villar del Humo (Cuenca) ${ }^{34}$. Dos arqueros de piernas en ángulo, bastante esquemáticos, se sitúan en la zona $A$ (fig. 7), pero el resto de motivos esquemáticos o semiesquemáticos se ubican en la $\mathrm{B}$. Muestran en algún caso señalados los dedos de los pies, peculiaridad que aparece también en otros yacimientos como en Los Órganos de Despeñaperros (Jaén) ${ }^{35}$ y en el abrigo de Solana de Las Covachas (Albacete) ${ }^{36}$. Otros portan un tocado a modo de penacho de plumas ${ }^{37}$ o en boina en forma de seta (fig. 9: 7), por lo

26 JORDA, F., y AlCACER, J., 1951, Las pinturas rupestres de Dos Aguas (Valencia), págs 11-12, fig. 4a, lám. III. Servicio de Investigación Prehistórica, Serie de Trabajos Varios, 15. Valencia 1951, págs. 11-12, fig. 4a, lám. III

2. Alonso, 1980, cit. nota 25, pág. 64, fig. 48.

28 BELTRAN, 1968, cit. nota 25, pág. 188, y figs. 119 y 122

29 Alonso, 1980, cit. nota 25, pág. 64, fig. 48.

30 RIPOLL, E., Los abrigos pintados de los alrededores de Santolea Teruel). Monografias de Arte Rupestre, Arte Levantino 1. Barcelona 1961, pág. 17, y plano III.

${ }^{31}$ Alonso, 1980, cit. nota 25, pág. 142, fig. 153.

32 Beltrán, A., Los abrigos pintados de la CaNaica del Calar y de La Fuente del Sabuco en el Sabinar (Murcia), Monografias Arqueológicas IX. Zaragoza 1972, pág. 67, fig. 62.

33 Acosta, P., La pintura rupestre esquemática en España. Memorias del Seminario de Prehistoria y Arqueologia. Salamanca 1968.

${ }^{34}$ Alonso et alii, 1982, cit. nota 8, págs. 133-137, figs. 4 y 9.

${ }^{35}$ González Navarrete, J., "Nuevas pinturas rupestres de Jaén. El Abrigo de los Órganos en Despeñaperros", Boletin del Instituto de Estudios Giennenses, XLVIII, 1971, pág. 9. lám IV.

36 Beltrán, 1968, cit. nota 22, pág. 250, fig. 154. Alonso, 1980, cit. nota 25, págs. 142-143. figs. 153 y 154 .

3) JORDÁ, F., «Los tocados de plumas en el arte rupestre levantino», Zephyrus, XXI-XXII, 1970-71, págs. $35-72$. 
que se paralelizan con abrigos de Valencia ${ }^{38}$ y Murcia ${ }^{39}$. Hileras de antropomorfos, al estilo de los dibujados en la zona B (fig. 9: 7) no son extraños en el arte esquemático, pues se encuentran en las pinturas del término de Villar del Humo (Cuenca), aunque en un estilo más abstracto, en los conjuntos de Marmalo $\mathrm{V}$ y Selva Pascuala ${ }^{40}$, asi como en algunas estaciones rupestres más distantes como Nuestra Señora del Castillo (Badajoz) ${ }^{41}$, Covacha de Monfragüe (Cáceres) ${ }^{42}$, Cholones Zagrilla (Córdoba) ${ }^{43}$. Piedras de La Cera y Cueva del Tío Labrador (Almería) ${ }^{44}$ y El Retamoso (Jaén) ${ }^{45}$. Es interesante la figura que encabeza este grupo, pues parece portar un cayado o báculo, de los que encontramos ejemplos en lugares tan dispares como Fuente de Los Molinos en Vélez Blanco (Almeria), Zarzalón (Salamanca) y Peña Tú y Fresnedo (Asturias) ${ }^{46}$.

Los antropomorfos de tipo cruciforme (fig. 9: 5) presentan gran cantidad de paralelos en el arte esquemático, por lo que obviamos su mención, aunque resaltamos el vacio interior de su cabeza, como el de gran cantidad de pictografias que se dibujan en Sesamo de Vega de Espinareda (León) ${ }^{47}$. Los ramiformes (fig. 9: 13) presentan paralelos cercanos en Dos Aguas (Valencia) ${ }^{48}$. Destaca además una representación al parecer humana con cabeza de animal o, al menos, con tocado de grandes cuernos (fig. 13: 3), lo que no es extraño en el arte de este tipo ${ }^{49}$ y que

\footnotetext{
${ }^{36}$ Monzonis, F., y Viñas, R., "Cinco nuevos abrigos con arte rupestre en la zona de Bicorp (Valencia)". Altamira Symposium, 1980, tigs. 9.1 y 9.7

${ }^{39}$ BreulL, $H$., "Les peintures rupestres de la Péninsule Ibérique VI: Les Abris Peintees du Monte Arabi, pres Yecla (Murcia)", L'Anthropologie, XXVI, 1915. Beltran, A., La Cueva de Los Grajos, sus pinturas rupestres en Cieza (Murcia). Monografias Arqueológicas, VI. Zaragoza 1969 .

40 Hernandez-PaCheCo, 1959, cit. nota 6, pág. 432, figs. 368 y 369. Alonso, 1984, cit. nota 9. fig. 10.

${ }^{41}$ Breuth. H., Les peintures rupestres schématiques de la Péninsule Ibérique, II, pl. XIX 10 y XX. Lagny 1933-35.

${ }_{42}$ Rivero de la Higuera, M. ${ }^{a}$ C.; "Nuevas estaciones de pintura rupestre esquemática en Extremadura», Zephyrus, XXIII-XXIV, 1972-73, págs. 288-291, fig. 1

${ }^{43}$ Bernier, J., y Fortea, F. J., "Nuevas pinturas rupestres esquemáticas en la provincia de Córdoba. Avance a su estudio", Zephyrus, XIX-XX, 1968-69, pág. 144, fig. 1

${ }^{44}$ BreUIL, 1933-35, cit. nota 41, vol. IV, pl. XXXIII Y XX, 1b.

45 BreulL, 1933-35, cit. nota 41, vol. Ill, fig. 22

46 JORDA, 1978, cit. nota 21, págs. 125-126.

4' Gutiérrez Gonzalez, J. A., y Avello Álvarez, J. L., Las pinturas rupestres esquemáticas de Sesamo, Vega de Espinareda (León). Monografias del Centro de Investigación y Museo de Altamira, 12, 1986

48 JORDA y ALCACER, 1951, cit nota 21, fig. 7. MONzONIS y VIÑAS, 1980, cit. nota 38, fig. 9.8.

${ }^{49}$ Ripoll, E., Las pinturas rupestres de La Gasulla (Castellón). Monografias de Arte Rupestre. Arte Levantino, 2. Barcelona 1963, lám. XI, fig. 14, y lám. XXV, fig. 2. ViNas. R., y Alonso, A., "L'abri de "Los Toros", las Bojadillas (Nerpio, Albacete)", Bulletin de la Société Préhistorique de l'Ariège, XXXIII, 1978, fig. 3, núm. 132. Dams, 1984, cit. nota 10, pág. 124. y
} 
algunos autores han interpretado como brujos y hechiceros ${ }^{50}$, símbolos del "dios toro» ${ }^{51}$ o incluso se le ha sugerido una significación astral o lunar ${ }^{52}$. Esta figura parece sostener con una mano un instrumento (?) y de la otra parten una serie de lineas ondulantes o serpentiformes, de dudosa interpretación. Se podria establecer un cierto paralelismo entre este motivo y la figura humana tocada con cuernos y armada con hoces de la cueva de Los Letreros en Vélez Blanco (Almería) ${ }^{53}$.

Las representaciones zoomorfas son de estilo naturalista y esquemático. Entre las primeras destaca la ausencia de toros, tan frecuentes en la estaciones rupestres de Villar del Humo y, por el contrario, el predominio del ciervo, animal raro en aquel conjunto. Los dos cérvidos que se conservan casi en su integridad presentan una cornamenta muy desarrollada, lo que no es común en el arte levantino, aunque se encuentran paralelos en Chimiachas y Arpán en el rio Vero en Huesca ${ }^{54}$ y en Solana de Las Covachas en Albacete en dos figuras de ciervos ${ }^{55} 0$, según Alonso ${ }^{56}$, de gamos, en el abrigo de Los Toros de Nerpio (Albacete) ${ }^{57}$. Si es habitual marcar las pezuñas en los animales, de lo que se encuentran múltiples ejemplos, los más cercanos en Villar del Humo (Cuenca) ${ }^{58}$ y en Dos Aguas (Valencia) ${ }^{59}$. La representación de cabra (fig. 5: 1), aparece también en La Peña del Escrito en Villar del Humo ${ }^{60}$, por indicar un ejemplo próximo. Se muestra aislada en la temática animalística de estilo naturalista por su extraña cornamenta, muy poco desarrollada y de trazo recto, y por la ausencia de orejas. Un ejemplo similar aparece representado en La Solana de Las Covachas (Albacete), y ante él Alonso ${ }^{61}$ se

fig. 117. Martinez Perello, M. ${ }^{a}$ I., "Un nuevo conjunto de pinturas esquemáticas de la Sierra del Pedroso (Peñalsordo y Capilla, Badajoz)", Ars Praehistorica, VIl-VIII (en prensa).

${ }^{50}$ RIPOLL, 1963, cit. nota 49, págs. 52 y 53 .

51 JORDÁ, 1975, cit. nota 7. JORDA, F., “¿Restos de un culto al toro en el Arte Rupestre Levantino?", Zephyrus, XXVI-XXVII, 1976, págs. 208-209.

52 Beltrán, A., Ensayo sobre el origen y significado del arte prehistórico. Zaragoza 1989 págs. 112-3.

${ }^{53}$ BreulL, 1933-35, cit. nota 42, vol. IV, pág. 12, fig. 3, pl. IX. Soria Lerma, M., y LoPEz PAYer, M. G., El arte rupestre en el Sureste de la Peninsula Ibérica. La Carolina (Jaén) 1989. pág. 33.

${ }_{54}$ Balldellou, $V$., "El arte rupestre postpaleolitico de la zona del rio Vero (Huesca)", Ars Praehistorica, III-IV, 1984-85, pág. 116 y fig. $5: 2$ y 5.

5s Beltrán, 1968, cit. nota 22, pág. 250, y fig. 155.

s6 Alonso, 1980, cit. nota 25, pág. 140, fig. 147, 151 y 158.

57 Viñas y ALONSO, 1978, cit. nota 49, pág. 107 y fig. 6.

SB DAMs, 1984, cit. nota 10, figs. 117-120.

59 JORDA y AlCACER, 1951, cit. nota 26, fig. 4.

go Hernandez-Pacheco, 1959, cit. nota 6 , págs. 422-3, fig. 358. Beltran, 1968, cit. nota 22, pág. 155.

6. Alonso, 1980, cit. nota 25, pág. 144 y fig. 155. 
plantea la posibilidad de que se trate de un cérvido y de que los pequeños cuernos puedan ser orejas.

En los zoomorfos esquemáticos se distingue un probable conjunto de cabras (fig. 13.1), motivos indeterminados y pectiniformes de tres, cuatro, cinco y diez trazos verticales inferiores. Los primeros constituyen un rebaño guiado por una figura humana, que interpretamos como el pastor. Escenas de rebaños se han documentado en Marmalo $V$ (Cuenca) ${ }^{62}$ pero sin estar asociada a una figura humana, además de en el Abrigo del Mirador (Soria) ${ }^{63}$ y en La Cuerda del Torilejo (Soria) ${ }^{64}$, en La Cañada de Marco (Teruel) ${ }^{65}$, La Graja de Jimena (Jaén) ${ }^{66}$ y en El Tajo de Las Figuras (Cádiz) ${ }^{67}$.

Pectiniformes semejantes a los de La Hoz de Vicente se localizan en Selva Pascuala, en Villar del Humo (Cuenca) ${ }^{68}$ y en el Abrigo II del Cinto de la Ventana en Dos Aguas (Valencia) ${ }^{69}$, por señalar los paralelos más próximos. Acosta señala también la presencia de cuadrúpedos esquemáticos en Doña Clotilde, La Vieja, Abrigo Sur de Carasoles del Bosque, Abrigos I y II de Cueva Negra de Meca, La Araña de Bicorp y Anear ${ }^{10}$, todos ellos relativamente cercanos a nuestro abrigo.

Como figuraciones abstractas se observan puntiformes, barras y serpentiformes. Los primeros se disponen en una línea de cinco unidades muy próximas entre sí (fig. 9.10), por lo que se diferencian de otras alineaciones, como las de Marmalo II y III (Cuenca) ${ }^{71}$, y se asemejan a los del extremo izquierdo de la Peña de Aldebarán (Cuenca) ${ }^{12}$.

Las barras es sin duda el tema más representado en el arte esquemático de la Peninsula Ibérica, e igualmente aparecen en el abrigo de La Hoz de Vicente, aunque no son muy numerosas. Se disponen tanto en horizontal como en vertical, aisladas o asociadas. En algún caso estos

62 Alonso, 1984, cit. nota 9, fig. 6 .

${ }^{63}$ Ortego, T., "Las estaciones de arte rupestre en el monte Valonsadero de Soria», Celtiberia, 2, 1951, págs. 275-305. Gomez Barrera, J. A., La Pintura Rupestre Esquemática en la Altimeseta Soriana. Soria 1982, fig. 22.

64 Gomez Barrera, 1982, cit. nota 63.

65 Ortego, T., "Una nueva estación de arte rupestre en el término de Alcaine (Teruel)", Simposio Internacional de Arte Rupestre. Barcelona 1968, pág. 155, fig. 10.

${ }^{65}$ Breull, 1933-35, cit. nota 41, vol. IV, pág. 5, lám. 2.

${ }^{67}$ Breuil, H., y Burkitt, M. C., Rock Paintings of Southern Andalusia. Oxford 1929, fig. 29.

68 DAMS, 1984, cit. nota 10, fig. 118.

69 JORDA y AlCACER, 1951, cit. nota 26, fig. 8.

10 Acosta, 1968, cit. nota 33, págs. 49-58, figs. 11 y 12 , mapas 8 y 9 .

$"$ Alonso, 1984, cit. nota 9, figs. 2 y 3.

PIÑON, 1986-87, cit. nota 11, fig. 1. 
trazos rectos parecen pertenecer a figuras de las que sólo quedan algunos restos.

Los serpentiformes se localizan aislados o en conjuntos y dispuestos en vertical u horizontal (fig. 9.10 y 14 y fig. 13.3), como, por ejemplo, en el Abrigo del Zuro de Bicorp (Valencia) ${ }^{73}$, su trazo no siempre es paralelo, como es frecuente en este tipo de representaciones. Hemos incluido estos motivos entre las abstracciones ya que no existe ningún elemento del contexto que permita interpretarlos como reptiles, agua o figuras humanas ${ }^{74}$, aunque habria que destacar la asociación de varios de estos elementos al antropomorfo tocado con cuernos (fig. 13.3).

\section{ALGUNAS CONSIDERACIONES RESPECTO A LAS REPRESENTACIONES PICTORICAS DEL ABRIGO DE LA HOZ DE VICENTE}

Partiendo de la base de que el arte que contiene el abrigo de La Hoz de Vicente representa una facies avanzada dentro del arte postpaleolítico, creemos que por sus caracteristicas cabe plantearse una serie de consideraciones.

Las sociedades prehistóricas del Noreste de la Meseta Sur no se presentan a lo largo de su historia homogéneas, sino más bien al contrario, muestran unos cambios trascendentales en sus sistemas socio-económicos, ideológicos, culturales y políticos. Las representaciones rupestres naturalistas, las más antiguas e infrapuestas en algún caso a las esquemáticas, comienzan con la aparición del modo de vida campesino. El primer contraste a subrayar será por tanto la representación mayoritaria (y en el abrigo de La Hoz de Vicente exclusiva) de actividades cinegéticas, en un momento en el que su importancia se halla en regresión con respecto a la agricultura y a la ganaderia, y el mayor detalle y naturalismo con el que se tratan las figuras animales frente a las humanas, realizadas con estilos tendentes a la idealización o a la esquematización. Quizá la explicación al problema del origen de este arte se halle en verlo, al igual que aduce Llavori, como «resultado de un conflicto de competencias territoriales, económicas y socioculturales insostenible, entre dos modos de

\footnotetext{
${ }^{13}$ MONZONIS y VIÑAS, 1980 , cit. nota 38 , fig. 3.

i4 Acosta, P., “Técnicas, estilo, temática y tipologia en la pintura rupestre esquemática hispana", Zephyrus, XXXVI, 1983, págs. 13-25.
} 
producción opuestos, definidos respectivamente por los grupos de cazadores-recolectores de filiación epipaleolitica por una parte y las comunidades productoras de alimentos por otra" ${ }^{75}$. Sin embargo, interpretar el arte rupestre sólo como expresión de un conflicto impide entender su continuidad y transformación hacia un mayor esquematismo en momentos posteriores como en la Edad del Bronce, en los que es dificil pensar en la perduración de sociedades cazadoras-recolectoras, por lo menos en el área que nos ocupa ${ }^{76}$. Es en este último período cuando las comunidades se sedentarizan y se agrupan en torno al mundo doméstico, lo que se correlaciona con los nuevos temas representados. En éstos, la figura humana cobra mayor importancia, aunque ahora se halla despojada de los trazos que la individualizaban, se concibe como un elemento más dentro de un grupo. En este «último" estilo aparecen por fin reflejadas las actividades económicas que, aún habiéndose practicado en la fase "previa», hasta este momento habian sido ignoradas en las representaciones rupestres.

En “ambos» momentos las pinturas rupestres se desenvuelven fuera del centro habitual de habitación, centrado éste en la aldea y en su área de captación más inmediata, donde se regulan las manifestaciones artísticas cotidianas ${ }^{77}$. Las representaciones se encuadran por lo tanto en un ámbito natural y salvaje, en el mundo externo. Este contexto no doméstico puede ser lá clave que justifique la falta aparente de orden, de gramática compositiva en el conjunto del panel.

El análisis del conjunto de la "obra» permite destacar que, pese a que los motivos en él inmersos provienen de sociedades que pintan en distin-

${ }^{75}$ Llavori, R., «El arte postpaleolítico levantino de la Peninsula lbérica. Una aproximación sociocultural al problema de sus orígenes", Ars Praehistorica, VII-VIII, 1988-1989, págs. 145156.

${ }_{76}$ En el NE. de la Meseta Sur parece que se adopta el policultivo de tipo mediterráneo en la Edad del Bronce, lo que correlaciona con un aumento progresivo de la tensión politica del Bronce Inicial al Medio, en el que se observa una jerarquización de los yacimientos, que decrece en el Bronce Final. El hecho de que la única actividad económica reconocible en los motivos esquemáticos de la mayoria de los abrigos rupestres y en concreto en La Hoz de Vicente sea el pastoreo, no significa el desconocimiento de la agricultura, puesto que la ausencia de representaciones de esta actividad podria estar relacionada con la imposibilidad de practicarla en la estrecha margen de la hoz del Cabriel en por lo menos 8 y $10 \mathrm{~km}$ rio arriba y abajo respectivamente. DiAz-AnDREu, M. La Edad del Bronce en el NE de la Submeseta Sur. Un análisis sobre el inicio de la complejidad social. Serie Tesis Doctorales. Universidad Complutense, 1990.

Proceso que se manifiesta por ejemplo, en la Edad del Cobre y Bronce, en la progresiva estandarización de las cerámicas y en los esquemas establecidos en su decoración en el Calcolítico Campaniforme, y en el Bronce Inicial y Final, o en la no decoración en el Bronce Medio. 
tos estilos, conviven en un mismo abrigo e incluso en un mismo panel "ambos" tipos de figuraciones, lo que parece indicar una pervivencia de un cierto sentido especial del lugar, sentido que a lo largo del tiempo sufre, sin duda, pequeñas pero continuas transformaciones. Asi a las representaciones iniciales naturalistas del abrigo de La Hoz de Vicente se añaden otras que tienen en cuenta para su ubicación, desarrollo y composición a las primeras. Se establece de esta manera una relación escénica entre ellas, multiplicándose las posibilidades de lectura, y por 10 tanto modificando el significado inicial del panel y de todo el lugar. Este proceso de interrelación parece influir incluso en el estilo de las tardias, que, condicionadas por la tradición artística anterior, abogan por un mayor dinamismo y detalle en las representaciones esquemáticas, un menor número de signos y abstracciones y un carácter narrativo más acentuado lo que es habitual en el arte esquemático. Las representaciones de La Hoz de Vicente son huella de la transformación gradual y continua de las sociedades en un lapso amplio de tiempo en el que se produce el cambio trascendental que supone el abandono del Paisaje Salvaje y la adopción del Paisaje Campesino ${ }^{78}$. $75-98$

${ }^{78}$ CRIAdo, F., "Megalitos, espacio, pensamiento", Trabajos de Prehistoria, 46, 1989, págs 